\title{
Occurrence of Cronobacter spp. in raw milk
}

\author{
Andreas Baumgartner $\cdot$ I. Niederhauser
}

Received: 23 February 2010 / Accepted: 17 March 2010/ Published online: 7 April 2010

(c) Springer Basel AG 2010

Cronobacter (Enterobacter sakazakii) is an opportunistic pathogen which can cause rare but serious infections in neonates. Neonatal infections were shown to be associated with dried infant milk formulae. Publications concerning the contamination of such foods with Cronobacter are numerous but there are almost no data available concerning the situation with raw milk.

We tested 875 bulk milk samples which were kindly given by three big milk processing companies. The samples represent milk producing farms from almost the entire territory of Switzerland and were recovered in the year 2007.

Milk samples were tested with a method which was used in a recent study and which was shown to have an excellent performance (Baumgartner et al. 2009). The applied test conditions were as follows: milk samples were defrosted at room temperature, hold at $40{ }^{\circ} \mathrm{C}$ for $20 \mathrm{~m}$ in a water bath and then transferred on a Stuart Rollermixer SRT9 (Bibby Scientific Ltd., UK) until analyzed. For bacteriological analysis, an aliquot of $10 \mathrm{~mL}$ milk was added to $90 \mathrm{~mL}$ of Cronobacter selective broth (CSB) and incubated overnight at $42{ }^{\circ} \mathrm{C}$ under aerobe conditions. Subsequently, single colonies were obtained by streaking $10 \mu \mathrm{l}$ of enrichment broth onto Enterobacter sakazakii isolation agar (ESIA) (AES Laboratoire; Bruz, France). Selective plates were incubated aerobically at $44{ }^{\circ} \mathrm{C}$ for $21 \pm 3 \mathrm{~h}$. Presumptive colonies (blue color) were sub-cultured on sheep blood agar

Dr. A. Baumgartner $(\bowtie) \cdot$ I. Niederhauser

Federal Office of Public Health, Section Microbiological and Biotechnological Risks, 3003 Bern, Switzerland

e-mail: andreas.baumgartner@bag.admin.ch
(BioMérieux 43041) and confirmed with the API ID 32E test system (BioMérieux 32400). As positive control, C. sakazakii DSM 4485 was used.

To our surprise, all the tested 875 bulk milk samples were negative for Cronobacter spp.! With a lower sample size, a similar observation was made by Lehner et al. (2010). These authors detected Cronobacter spp. in 172 samples of milk powder but not in 100 raw milk samples representing tank milk samples from approximately 1,240 sampling points in Switzerland.

Our finding, based on a very large sample size, supports the conclusion of Lehner et al. that milk cannot be a relevant source for Cronobacter spp. and that contaminations of milk powders are originating most likely from the environment. In foods of plant origin for example, Cronobacter is highly prevalent as we could show with the analysis of fresh sprouts, herbs and salads and dried spices and herbs (Baumgartner et al. 2009).

\section{References}

Baumgartner A, Grand M, Liniger M, Iversen C (2009) Detection and frequency of Cronobacter spp. (Enterobacter sakazakii) in different categories of ready-to-eat foods other than infant formula. Int J Food Miccrobiol 136: 189-192

Lehner A, Fricker-Feer C, Gschwend K, Stephan R (2010) Identification of Enterobacteriaceae and Cronobacter spp. in raw milk, milk concentrate and milk powder: prevalence and genotyping. Arch Lebensmittelhyg 61:22-26 